\title{
POLYDOPAMINE COATED Si NANOPARTICLES ALLOW fOr IMPROVED MECHANICAL and ELECTROCHEMICAL STABILITY
}

\author{
Utkarsh Ahuja $^{\mathrm{a}}$, Bo Wang ${ }^{\mathrm{a}}$, Pu Hu $\mathrm{b}^{\mathrm{b} *}$, Julien Rethore ${ }^{\mathrm{c}}$ and Katerina E. Aifantis ${ }^{\mathrm{a}^{*}}$ \\ ${ }^{a}$ Department of Mechanical and Aerospace Engineering, University of Florida, Gainesville, 32603, USA \\ ${ }^{b}$ Hubei Key Laboratory of Plasma Chemistry and Advanced Materials, School of Materials Science and \\ Engineering, Wuhan Institute of Technology, Wuhan, 430205, China \\ ${ }^{c}$ Research Institute in Civil and Mechanical Engineering (Gem), CNRS UMR 6183 CNRS/Ecole Centrale \\ de Nantes/Université de Nantes, F-44 321 Nantes, France
}

\begin{abstract}
Numerous experiments suggest that the capacity decay of Silicon ( $\mathrm{Si}$ ) porous electrodes is related to the significant fracture experienced during the lithiation/de-lithiation process. In this work modeling and surface engineering of nanosized Si is employed to synthesize nanocomposites with enhanced mechanical and electrochemical stability. Initially, a multiphysics model is applied to predict the size of Si particles that limit damage formation. The model is experimentally verified against scanning electron microscopy (SEM), which shows the fracture of Si microparticles after the first and second cycles. Particles less than $100 \mathrm{~nm}$ are predicted to be mechanically stable, and to further increase stability, a facile one-step in-situ polymerization process is used to synthesize $\mathrm{Si} /$ polydopamine ( $\mathrm{Si} / \mathrm{DPA}$ ) nanocomposites, in which $\sim 2 \mathrm{~nm}$ of polydopamine (DPA) uniformly coats the surface of the Si. The as-prepared electrodes exhibit higher capacity than previously reported Si/DPA composites: $2000 \mathrm{mAh} \mathrm{g}^{-1}$ at $\sim 700 \mathrm{~mA} \mathrm{~g}^{-1}$, with a $66 \%$ retention after 100 cycles. A $15 \%$ higher capacity retention is observed herein for the Si/DPA nanocomposite electrode compared with the pure nano-Si electrode. The enhanced capacity retention of the nanocomposite electrode can be attributed to the engineered polymeric layer which can alter the stresses experienced by the Si surface during lithiation and enhances adhesion within the nanocomposite electrode.
\end{abstract}

Keywords: Si anode; Si/polymer; fracture; phase field; Li-ion; electron microscopy 


\section{Introduction}

Use of Silicon $(\mathrm{Si})$ as an anode for lithium-ion batteries $(\mathrm{LiB})$ has received widespread attention because of its large theoretical specific gravimetric capacity of $3579 \mathrm{mAh} \mathrm{g}^{-1}$ [1] caused by formation of $\mathrm{Li}_{15} \mathrm{Si}_{4}$ alloy at room temperature [1]. However, upon lithiation Si active particles undergo a volume expansion of up to $320-400 \%$ [2-4] impacting the mechanical integrity of the electrode, and leading to pulverization of $\mathrm{Si}$ [5-7]. This severe fracture was first observed by performing scanning electron microscopy (SEM) on Si thin film electrodes after cycling [8]. Since then, in-situ lithiation in transmission electron microscopy (TEM) has been used to document the fracture that Si nanoparticles experience [9-11]. It is, therefore, assumed that the capacity fade in Si porous electrodes is due to fracture of the Si particles, which is expected to be more severe as the particle size increases. Despite this, fracture for individual Si micro-particles has not been rigorously documented until now. In [12] SEM images showed fracture of porous electrodes after cycling but electron dispersive spectroscopy (EDS) was not performed to indicate if the cracks occurred in the Si or binder. Particularly, the expected fracture in porous electrodes results in a loss of electronic pathways, as wells as delamination of the active material from the conductive matrix and current collector [13]. The new fractured surfaces allow for additional reduction of the electrolyte resulting in the formation of a solid electrolyte interphase (SEI) layer that is unstable and continues to grow during cycling, consuming $\mathrm{Li}^{+}$and electrolyte [14]. As a result, significant capacity fade occurs in Si electrodes.

The most common approach to increase the mechanical stability of $\mathrm{Si}$ is to use nanostructured $\mathrm{Si}$ particles $[9,15]$, as well as employing nanostructured morphologies such as solid core-shell, hollow coreshell, nanotubes, and nanowires to increase capacity retention [16-18]. The effectiveness of different binders in combination with Si has also been examined. Specifically, Carboxymethyl cellulose (CMC), Polyacrylic acid (PAA), Polyvinyl alcohol (PVA), alginate, gels or combinations of these have shown to buffer the Si expansion during lithiation due to their high yield strength and self-healing characteristics [19-22]. 
Some promising binder materials chemistries and configurations are the following.-Impregnated gels (which act as binders) with Si nanoparticles $(30-100 \mathrm{~nm})$ resulted in a capacity retention of $2283 \mathrm{mAh}$ $\mathrm{g}^{-1}$ after 100 cycles (at $400 \mathrm{~mA} \mathrm{~g}^{-1}$ ) [23]. A three-dimensional cross-linked polymeric binder (sodium-CMC, $\mathrm{Na}-\mathrm{CMC}$ and PAA) with nanosized Si powder (less than $100 \mathrm{~nm}$ ) and Super P carbon allowed for a reversible capacity that was over $2000 \mathrm{mAh} \mathrm{g}^{-1}$ after 100 cycles (at $300 \mathrm{~mA} \mathrm{~g}^{-1}$ ). The authors attributed the improved capacity retention to the cross-linked binders which exhibited a high mechanical resistance to permanent deformation. In these studies, cross-linking of binders yielded a good capacity retention however, the rate of cycling was low (300-400 $\left.\mathrm{mA} \mathrm{g}^{-1}\right)$.

Another effective approach for improving the performance of Si is to encapsulate or coat [24-26] the Si surface with polymers such as polypyrrole, tannic acid, polyaniline can also increase the cycling stability $[27,28]$. Such coatings help improve the electronic conductivity, increase Li-ion diffusivity and increase binding due to cross-linking of otherwise linear binders. Coating Si particles (50-200 nm) with a thin layer of polydopamine (DPA) to form a three-dimensional cross-linked system with PAA binder yielded a capacity of $1800 \mathrm{mAh} \mathrm{g}^{-1}$ after 100 cycles [29]. The authors performed characterization (Fouriertransform infrared spectroscopy/FTIR, X-ray photoelectron spectroscopy/XPS) to confirm the presence of DPA and its interaction with the PAA binder. They confirmed that the better capacity retention was due to the robustness of the electrode system but electron microscopy after cycling was not performed. An improved capacity retention of $1200 \mathrm{mAh} \mathrm{g}^{-1}$ after 100 cycles (at $840 \mathrm{~mA} \mathrm{~g}^{-1}$ ) was also obtained by using graphene oxide and DPA to coat $\mathrm{Si}$ [30]. Characterization of the as-prepared samples showed that agglomeration was prevented, and it was assumed that the flexible polymer coatings helped buffer the volume expansion.

Various mechanical models have been developed in order to capture the mechanical stability of Si electrodes during electrochemical cycling [31-36], with the most promising one being presented in [36], as it was able to use phase field modelling to capture the long-standing fracture patterns that develop in Si thin films. In this study, we extend this multiphysics model for the geometry of spherical Si particles coated 
with a polymer, in order to predict the Si particle size that will limit fracture. Based on the insight provided by the model Si nanoparticles were coated with a DPA thin film by a facile in-situ polymerization approach to strengthen the mechanical stability of the Si anode. The as-obtained Si/DPA nanocomposites showed improved capacity retention and structural/mechanical stability over uncoated Si electrodes.

\section{Experimental}

\section{Materials}

Si nanoparticles ( $\mathrm{Si}$, crystalline, APS $\leq 50 \mathrm{~nm}, 98 \%$ ), acetylene black and dopamine monomer (Dopamine Hydrochloride, 99\%) were purchased from Alfa Aesar. Si microparticles were procured from US Research Nanomaterials, Inc. Polyacrylic acid (PAA, 35 wt. \% in $\mathrm{H}_{2} \mathrm{O}$ ) was obtained from Aldrich Chemistry. Tris$\mathrm{HCl}(1 \mathrm{M}, \mathrm{pH} 8.5)$ was obtained from Teknova, and $\mathrm{Cu}$ foil was obtained from MTI corporation. Lithium hexafluorophosphate $\left(\mathrm{LiPF}_{6}\right)$ in ethylene carbonate and dimethyl carbonate $(\mathrm{EC} / \mathrm{DEC}=50 / 50 \mathrm{v} / \mathrm{v})$ was purchased from Sigma fisher. Dimethyl carbonate was purchased from Sigma Aldrich. Deionized water (reagent grade) was used for all aqueous preparations. All chemicals used were of analytical grade and were used as received without further purification.

\section{Synthesis of Si/DPA nanocomposite}

Si nanoparticles was dispersed in ethanol and subjected to ultrasonication for an hour to form a uniform suspension. $40 \mathrm{mM}$ tris buffer and dopamine- $\mathrm{HCl}$ (monomer) were added to the suspension and stirred for 24 hours at room temperature. The resultant suspension was centrifuged at $12100 \mathrm{rpm}$ for 15 minutes and oven dried at $60^{\circ} \mathrm{C}$ overnight to obtain Si/DPA nanocomposites. Mass fraction of Si:monomer was changed from 1:1 to 1:2, however, this did not yield appreciable change in the thickness of the polymer coating on Si. Repeated polymerization cycles on Si/DPA nanocomposites yielded large polymer particles de-attached from the nanocomposite, thereby decreasing active mass loading. Hence repeated polymerization cycles were not investigated any further. 


\section{Electrode Fabrication}

Conventional porous electrodes were fabricated by mixing the active material with binder and carbon. Particularly, an aqueous slurry was made by mixing $60 \mathrm{wt} \%$ active material (Si nanoparticles or Si/DPA nanocomposite), $30 \mathrm{wt} \%$ acetylene black as the conductive agent, and $10 \mathrm{wt} \%$ Polyacrylic Acid (PAA) as the binder. The obtained slurry was then coated on a $\mathrm{Cu}$ foil current collector via doctor blading to maintain a uniform electrode thickness. This fabrication method of electrodes results in inherent porosity, and that is why they are termed "porous". The porosity is beneficial as it offers empty/void space for the active material to expand into, but also allows for easy diffusion of electrolyte and faster reaction kinetics. The electrode was dried at $60^{\circ} \mathrm{C}$ for 8 hours and then cut into disks of $\phi 12 \mathrm{~mm}$. The cut electrodes were baked at $120^{\circ} \mathrm{C}$ for 24 hours under vacuum. An areal active mass loading of $0.5-0.8 \mathrm{mg} \mathrm{cm}^{-2}$ was achieved by the doctor blading process. Hereafter, the electrodes made using Si nanoparticles and Si/DPA nanocomposite as the active materials will be referred to as $\mathrm{Si}$ electrode and $\mathrm{Si} / \mathrm{DPA}$ electrode respectively. Micron sized $\mathrm{Si}$ particles were also used to fabricate electrodes using the same technique and will be referred to as microscale Si electrode.

\section{Electrochemical Testing}

The electrochemical properties of the $\mathrm{Si}$ and Si/DPA electrodes were investigated using CR2032 cells assembled in a Mbraun UniLab Glovebox using Argon as the working gas. Pure Lithium disks were used as the counter electrode and $1.0 \mathrm{M} \mathrm{LiPF}_{6}$ in $\mathrm{EC} / \mathrm{DEC}(1: 1, \mathrm{v} / \mathrm{v})$ with $5 \%$ Fluoroethylene Carbonate (FEC) as additive was used as the electrolyte. A fiberglass separator (Fisherbrand, Filter circles G6) was placed between the electrodes. A CT2001A Landt Instruments battery tester was used to cycle the cells through a constant current (CC) protocol between $0.02 \mathrm{~V}$ and $2 \mathrm{~V}$. Electrochemical Impedance Spectroscopy (EIS) data was obtained on a Gamry Instruments Interface 1010E potentiostat.

\section{Material characterization}


Thermogravimetric analysis (TGA, Q5000) was used to determine the mass ratio of Si to DPA polymer in the $\mathrm{Si} / \mathrm{DPA}$ nanocomposite. The analysis was performed in pure $\mathrm{N}_{2}$ atmosphere starting from room temperature until $800{ }^{\circ} \mathrm{C}$ at $10{ }^{\circ} \mathrm{C}$ per minute.

Fourier-Transform Infrared Spectroscopy (FTIR, ThermoFisher iS50) was performed to determine if the DPA polymer fully coated the Si particles.

The initial microstructure and the changes in morphology and structure during electrochemical cycling were studied with scanning electron Microscopy/SEM (TESCAN MIRA3 and TESCAN VEGA3) and transmission electron microscopy/TEM (Aberration Corrected S/TEM JEM-ARM200cF and FEI Tecnai F20 S/TEM) equipped with Energy dispersive X-ray spectroscopy (EDS). Conductive carbon tape was used to adhere the samples onto the stub holder in the SEM. Copper and gold grids with amorphous carbon films were used for TEM imaging. Samples were plasma cleaned before imaging under the TEM, when required. In performing post-cycling microscopy, the cells were opened in the glove box, and the electrodes were washed with DMC (dimethyl carbonate) to remove the electrolyte salt. For TEM, some powder from the electrodes was scraped on the grid.

\section{Multiscale Modeling}

To quantify the damage evolution of the electrode particle due to Li-ion diffusion, a multi-physics model is adopted. The model used is a phase field damage model coupled with the diffusion equation with a nonlinear concentration-dependent diffusivity [36]. The phase field model treats crack boundaries as continuous interfaces, and it doesn't need to explicitly track the crack front. By employing the phase field method for modeling fracture and then using a history variable, the problem can be recast as two linear problems instead of solving a non-linear two-field problem for the mechanical equilibrium and damage evolution, which reduces the complexity greatly. Herein we employ the model of [36], as it was successfully applied to capture the long-standing observation of fracture in Si thin film electrodes. Even though the 
model does not account for plasticity, which can occur in Si during the charge-discharge cycle, it is a good first approximation to obtain a measure of the effect of particle size. The Li-ion diffusion is assumed to be isotropic and the diffusion equation is given by Fick's law as:

$$
\frac{\partial c}{\partial t}=D \nabla^{2} c
$$

where the diffusivity parameter $D$ is given by $D=D_{0}\left(\frac{1}{1-c}-2 \times 1.95 \times c\right)$ and $c$ denotes the normalized Li-ion concentration, which ranges between 0 and 1, corresponding to full de-insertion (pure $\mathrm{Si}$ ) and fullinsertion $\left(\mathrm{Li}_{3.75} \mathrm{Si}\right)$, respectively. Under normal experimental conditions $\mathrm{Li}_{3.75} \mathrm{Si}$ is the fully lithiated state. $\mathrm{Li}_{4.4} \mathrm{Si}$, can form only under certain temperature conditions (688K-750 K) [37-39]. Here a non-linear diffusivity is used to produce a sharp phase boundary, accounting for the saturation in Li-ions during insertion when $c$ reaches $1 . D_{0}$ is the initial diffusivity, when no Li-ions are present in the $\mathrm{Si}$, i.e $c=0$. By using this nonlinear concentration-dependent diffusivity, the initial change of $D$ with respect to $c$ is very small and when $c$ approaches 1 then $D$ tends to infinity, and this prevents $c>1$. For Li de-insertion, the same expression is adopted but replacing $c$ with $1-c$. The small strain formulation is adopted. The total strain $\boldsymbol{\varepsilon}_{t}$ of the material is decomposed as the sum of the Li-concentration induced strain $\boldsymbol{\varepsilon}_{c}$ and the elastic strain $\boldsymbol{\varepsilon}_{e}$ :

$\boldsymbol{\varepsilon}_{t}=\frac{1}{2}\left\{(\nabla \boldsymbol{u})+(\nabla \boldsymbol{u})^{T}\right\}=\boldsymbol{\varepsilon}_{e}+\boldsymbol{\varepsilon}_{c}$

where

$\boldsymbol{\varepsilon}_{c}=\alpha c \boldsymbol{I}$.

It is seen that $\boldsymbol{\varepsilon}_{c}$ is defined similarly as the thermal strain analogy, where $\alpha$ is the dilatation coefficient that denotes the strain induced for full Li-insertion $(c=1)$ and a negative $\alpha$ is adopted for de-insertion. $I$ is the identity matrix and $\boldsymbol{u}$ is the displacement field. 
The equilibrium equation is

$\nabla \cdot \boldsymbol{\sigma}=\mathbf{0}$

where $\boldsymbol{\sigma}$ is the Cauchy stress tensor. The governing equation of damage evolution is given by [36]:

$G_{c}\left(-l^{2} \Delta d+d\right)=2 l(1-d) H$

where the phase field variable $d$ can only grow from 0 to 1 ( $d=0$ for unbroken/intact state, $d=1$ for fully broken/damaged state), $G_{c}$ is Griffith's critical energy release rate for unstable crack/damage extension, and $l$ is an internal length characteristic of the material. $H$ is a history variable representing the maximum elastic strain energy density and is given by [36]:

$$
\mathbf{H}=\max _{\tau \in[0, t]} \psi^{+}(\mathbf{u}, \tau)^{\prime}
$$

where $\psi^{+}$is the strain energy density associated to the extensive part of the elastic deformation, given by [36]:

$\psi^{+}=\frac{1}{2} k\left(\left\langle\operatorname{tr}\left(\boldsymbol{\varepsilon}_{e}\right)\right\rangle_{+}\right)^{2}+\mu\left\{\operatorname{tr}\left(\left(\boldsymbol{\varepsilon}_{e}\right)^{2}\right)-\frac{1}{3}\left(\operatorname{tr}\left(\boldsymbol{\varepsilon}_{e}\right)\right)^{2}\right\}$

where $\lambda$ and $\mu$ are Lame's constants, $\langle x\rangle_{ \pm}=\frac{1}{2}(x \pm|x|)$ are the positive and negative part of $x$, and $k=\lambda+\frac{2}{3} \mu$ is the bulk modulus. The Cauchy stress tensor is given by [36]:

$\boldsymbol{\sigma}=\left\{(1-d)^{2}\left\langle\operatorname{tr}\left(\boldsymbol{\varepsilon}_{e}\right)\right\rangle_{+}+\left\langle\operatorname{tr}\left(\boldsymbol{\varepsilon}_{e}\right)\right\rangle_{-}\right\} k \mathbf{I}+2 \mu(1-d)^{2}\left\{\boldsymbol{\varepsilon}_{e}-\frac{1}{3} \operatorname{tr}\left(\boldsymbol{\varepsilon}_{e}\right) \mathbf{I}\right\}$

The governing equations can be solved by applying appropriate initial and boundary conditions. Since the model is decoupled, the displacement field can be found after the diffusion equation is solved. Consequently, the damage evolution can be also obtained. This formulation allows for solving the mechanical equilibrium and damage evolution respectively. From a computational viewpoint, this is 
extremely convenient, and it reduces the complexity. To be specific, the concentration can be easily obtained by solving the diffusion equation (Eq. (1)). Once we get this concentration field, we can substitute into the mechanical equilibrium equation (Eq.(4)). The damage field and displacement from the previous time step are known, and as a result, the only unknown in the mechanical equilibrium equation is the displacement field. It should be noted that the elastic part of the strain equals to the total strain (symmetric gradient of displacement field) minus the strain due to lithiation (linearly dependent on the concentration field); as shown in Eq. (2) and Eq. (3). When the displacement field is obtained, the governing equation for the damage (Eq. (5)) can be solved subsequently.

\section{Results and discussion}

\subsection{Multiscale model}

Most spherical particles don't come into direct contact with current collectors. For freestanding particles, large homogeneous volume expansion and contraction of Si alone cannot cause severe cracks according to the model. Instead, it's the abrupt change in lithium concentration that gives rise to a mismatch strain between the lithiated $\mathrm{Si}$ and pure Si phases. As a result, crack formation and irreversible capacity fade occur. The model of Section 3 is implemented in 2D and in order to capture the geometry of porous electrodes, 5 larger Si particles with a diameter of $0.95 \mu \mathrm{m}$ and 4 smaller particles with a diameter of 450 $\mathrm{nm}$ are embedded in a square polymer binder with a side length of $5550 \mathrm{~nm}$. The initial conditions are $c=$ 0 and $c=1$, for Li-insertion and de-insertion respectively. The boundary conditions for this system are a constant flux of Li-ions $\left(1.4 \mathrm{~nm} \mathrm{~ms}^{-1}\right)$ [36] on the outer surface of the electrode and fixed displacement boundary conditions [40] for the exterior surfaces of the polymer. It takes about 10 hours to finish the full $\mathrm{Li}$ insertion; as a result, the C-rate is $0.1 \mathrm{C}$. The Young's modulus of the Si electrode and polymer is 130 GPa and 2 GPa respectively, and the Poisson's ratio is 0.2 and 0.34 respectively [36,41]. The purpose of the model here was to capture fracture of the $\mathrm{Si}$, not the polymer, and therefore we employ the modulus

for PVDF as an approximation. The initial diffusion coefficient is $25 \mathrm{~nm}^{2} \mathrm{~s}^{-1}$ [36] inside the Si particle and 
the value in the polymer is taken to be 1000 times as in the active material. By doing so, it ensures that the ion diffusion inside the polymer is immediate. The expansion coefficient $\alpha$ of the Si electrode is taken to be 0.3 [36]. As for the binder, it is assumed to be zero, since it could not host Li-ions [40]. The energy release rate for the Si electrode and internal length are $10 \mathrm{~J} \mathrm{~m}^{-2}$ [42] and $10 \mathrm{~nm}$ respectively. We assume that the polymer does not fracture, and for the model to capture this, the energy release rate for the polymer is taken to be 1000 times higher than that of $\mathrm{Si}$. The governing equations are solved in the open-source software FEniCS, by employing an unstructured mesh that has a size approximately $1 / 100$ of the side length of the polymer. Damage profiles after insertion and de-insertion are as shown in Fig. 1. Based on the simulation results, it is seen that cracks begin to initiate in the $0.95 \mu \mathrm{m}$ particles after complete insertion and continue to propagate during the de-insertion process cutting the particles in half.

To investigate further into the relationships between particle size and its fracture pattern, more simulations are performed by changing only the Si particle size in the simulations. Our results show that fracture is always observed until a critical size of $95 \mathrm{~nm}$, below which crack did not occur. This is illustrated in the damage profile shown in Fig. 1 (c)-(d) where the diameter of the larger particles is $95 \mathrm{~nm}$ and that of the smaller ones is $45 \mathrm{~nm}$. Therefore, this model can capture size effects and the results are consistent with in-situ TEM experiments which have shown that Si particles less than $100 \mathrm{~nm}$ do not fracture upon electrochemical cycling [9]. It should be noted that the particle distribution in addition to size plays a role in the fracture pattern since and this will be illustrated in a future publication.

\subsection{Microscale Si porous electrodes}

In order to test if the model predictions of Fig. 1 can capture the mechanical stability of micron-size $\mathrm{Si}$ particles during lithiation, porous electrodes comprised with microscale Si particles are tested. Fig. 2(a) shows the voltage profile of the microscale Si electrode at $0.2 \mathrm{C}$. The initial discharge profile demonstrates a flat voltage plateau corresponding to the formation of $\mathrm{Li}-\mathrm{Si}$ alloy during lithiation. The microscale $\mathrm{Si}$ 
electrode displays an initial discharge and charge capacity of $\sim 3390 \mathrm{mAh} \mathrm{g}^{-1}$ (corresponding to $\mathrm{Li}_{3.54} \mathrm{Si}$ ) and $\sim 3050 \mathrm{mAh} \mathrm{g}^{-1}$ respectively, corresponding to an initial coulombic efficiency (C.E.) of $90 \%$. Subsequent cycles yielded C.E. of $98.2 \%$ and $98.4 \%$. Fig. 2(b) shows that the discharge capacity decays with continuous cycling.

In order to understand the mechanisms that give rise to this capacity decay, cells are opened after one electrochemical cycle and SEM with EDS is performed. Fig. 3 reveals as is predicted by the phase field model (Fig. 1), that the micron-size Si particles fracture in half after one electrochemical cycle and the crack begins to cleave the particle after two electrochemical cycles. Such distinct fracture of an individual Si microparticle has not been documented with both SEM and EDS after lithiation. In Fig. S2 a SEM image of a micron scale Si particle after 20 cycles illustrates that the cracks grow with continuous cycling and begin to split up the particle. The particle enclosed with the dashed circle has dimensions smaller than $1 \mu \mathrm{m}$, suggesting that this Si piece broke off the parent Si particle after multiple cycles.

\subsection{Structure of $\mathrm{Si}$ and Si/DPA nanocomposite}

Fig. 4(a) is an illustration of the Si/DPA nanocomposite obtained after centrifugation and its interaction with polyacrylic acid. Aggregation of a few Si nanoparticles during polymerization can yield grouped $\mathrm{Si}$ nanoparticles within the polydopamine layer.

Fig. 4(b) shows the FTIR spectra of the Si/DPA nanocomposite along with the reference spectra of pure Si nanoparticles and pure DPA polymer. A broad absorption band between $3200-3500 \mathrm{~cm}^{-1}$ is observed in the spectra of $\mathrm{Si}, \mathrm{Si} / \mathrm{DPA}$ and DPA. This band can be assigned to the stretching modes of O-H and N-H bonds in Si/DPA and DPA spectra, and just the stretching mode of O-H bonds in the Si spectra [43-46]. The peaks near $1515 \mathrm{~cm}^{-1}$ in the spectra of $\mathrm{Si} / \mathrm{DPA}$ and DPA can be ascribed to the stretching vibration of the $\mathrm{C}=\mathrm{C}$ bonds in the indole structure $[44,46]$. The weak shoulder at $1723 \mathrm{~cm}^{-1}$ for both Si/DPA and DPA can be indexed to the $\mathrm{C}=\mathrm{O}$ bonds in the structure [45]. The low intensity of the abovementioned shoulder and presence of the absorption peak at $1515 \mathrm{~cm}^{-1}$ confirms that the polymerization of dopamine monomer 
occurred [45,47]. The strong peak at $1223 \mathrm{~cm}^{-1}$ for both $\mathrm{Si}$ and $\mathrm{Si} / \mathrm{DPA}$ can be assigned to the stretching mode of Si-O-Si bonds [43]. The peaks at $2101 \mathrm{~cm}^{-1}$ and $2259 \mathrm{~cm}^{-1}$ correspond to the stretching mode of Si-Si-H and OSi-H, respectively $[43,48]$. Finally, the shoulders at $969 \mathrm{~cm}^{-1}$ and $886 \mathrm{~cm}^{-1}$ can be ascribed to the stretching of $\mathrm{SiO}$ and OSi-H deformation, respectively [43]. TGA as shown in Fig. 4(c) is performed on the Si/DPA nanocomposite to ascertain the exact mass ratio of the Si to the polymer. The data alludes to the existence of about $10.4 \%$ polymer by weight in the Si/DPA nanocomposite. TGA of pure Si nanoparticles displays very minimal weight loss. TEM images as shown in Fig. 4(d) and 4(e) of Si/DPA nanocomposite confirm that the polymer forms a thin coating on the Si active particles. The thickness of the coating as denoted by red lines/arrows varies between $1.5-3 \mathrm{~nm}$.

\subsection{Electrochemical performance of nanoscale $\mathrm{Si}$ and $\mathrm{Si} / \mathrm{DPA}$ electrodes}

The voltage profiles of the pure nano $\mathrm{Si}$ and $\mathrm{Si} / \mathrm{DPA}$ electrodes at $0.2 \mathrm{C}$ are shown in Fig. 5(a)-(b), indicating similar behavior and characteristics. Both electrodes during the first discharge exhibit a flat voltage plateau observed at $50 \mathrm{mV}$ which is consistent with the formation of Li-Si [1] alloy during lithiation. The flat portion of the plot describes an interface-limited step wherein crystalline $\mathrm{Si}$ converts to amorphous $\mathrm{Li}_{\mathrm{x}} \mathrm{Si}$ [49]. Subsequent cycling did not show the same plateau, instead lithiation and delithiation occur at higher potentials and are denoted by a finite voltage slope [49].

The Si electrode demonstrates an initial discharge and charge capacity of $\sim 3400 \mathrm{mAh} \mathrm{g}^{-1}$ and $\sim 2900$ $\mathrm{mAh} \mathrm{g}^{-1}$ respectively, corresponding to an initial C.E. of $85 \%$. Further discharge/charge cycles showcase a large drop in the discharge capacity. Both, the decrease in discharge capacity and low initial C.E., can be attributed to the formation of SEI. The Si/DPA electrode exhibits lower initial discharge and charge capacities of $\sim 3125 \mathrm{mAh} \mathrm{g}^{-1}$ and $\sim 2675 \mathrm{mAh} \mathrm{g}^{-1}$, however, the C.E. remains the same as for pure $\mathrm{Si}$, at $85 \%$. However, a minimal discharge capacity loss is observed in the Si/DPA electrode after the $1^{\text {st }}$ cycle. Given that the initial voltage plateau is identical for both electrodes, no change in the mechanism of the 
lithiation/de-lithiation is indicated. Prolonged cycling of the electrodes is necessary to divulge the efficacy of the polymer coating.

Regarding long term cycling, the nano Si electrode cycled in Fig. 5(c) displays an initial discharge capacity and C.E. of $3500 \mathrm{mAh} \mathrm{g}^{-1}$ and $89.8 \%$ respectively. After 100 cycles, the discharge capacity decays to $1550 \mathrm{mAh} \mathrm{g}^{-1}$, and the C.E is $99.3 \%$. The Si/DPA nanocomposite electrode exhibits an initial discharge capacity and C.E. of $3000 \mathrm{mAh} \mathrm{g}^{-1}$ and 84\%, respectively. Upon continued cycling, the discharge capacity after 100 cycles decays to $1971 \mathrm{mAh} \mathrm{g}^{-1}$ and the C.E. approaches $100 \%$. An increase in the C.E. towards $100 \%$ alludes to the existence of a stable SEI layer. After 100 cycles, a discharge capacity retention of $66 \%$ is observed and the discharge capacity begins to plateau thereby exhibiting very little capacity fade. It is seen that a significantly better capacity retention is observed when a thin polymeric layer covers the $\mathrm{Si}$ active particle.

Fig. 5(d) compares the cycling performance of the electrodes at $1 \mathrm{C}$. It is observed that the Si/DPA electrode shows a rapid initial capacity fade but is able to retain an almost constant capacity of $600 \mathrm{mAh}^{-}$

${ }^{1}$ for 200 cycles. The nano Si electrode initially shows a high capacity, but it continuously decays over the entire cycling period. The initial lower capacity of the Si/DPA electrode may be due to the partially insulating nature of DPA. However, DPA prevents further capacity fade as is experienced by the pure nano Si electrode. In the supplementary information Fig. S3 illustrates the performance of a porous electrode with a composition of 80:10:10 for Si/DPA:C:Binder instead of 60:30:10. It is interesting that even though the Si content is higher the resulting capacity is lower, which shows that conductivity of the electrode is a key factor affecting its performance.

\subsection{Morphological changes of nanoscale $\mathrm{Si}$ and $\mathrm{Si} / \mathrm{DPA}$ electrodes}

A significant capacity decay of $56 \%$ and $34 \%$ is observed for the nanoscale and Si/DPA electrodes even though the Si particles are $\sim 50 \mathrm{~nm}$, and based on the model predictions fracture is not expected. To 
verify that the Si particles are able to retain their structural stability TEM is performed after the $1^{\text {st }}$ and $10^{\text {th }}$ cycles.

TEM imaging in Fig. 6 confirms that the Si nanoparticles did not fracture upon continuous cycling. The contrast in the TEM image of Fig. 6(c) shows the existence of a core-shell structure in a Si nanoparticle after 10 cycles. In-situ experiments have shown that when this kind of contrast in observed in TEM, it is due to a crystalline $\mathrm{Si}$ core and an amorphous $\mathrm{Li}_{\mathrm{x}} \mathrm{Si}$ shell [10]. Similar observations and the existence of an atomically sharp interphase $(\sim 1 \mathrm{~nm})$ between the crystalline and amorphous phases have been made during in-situ lithiation of $\mathrm{Si}[50,51]$. Existence of a core-shell structure alludes to an incomplete lithiation reaction [10], which can be caused by the detachment of Si from the current collector or the surrounding conductive $\mathrm{C}$; this can occur during the volume changes upon cycling. Such detachment of Si results in a loss of electronic pathway leading to an abrupt end of the reaction.

TEM images of the Si/DPA active material also did not show fracture; however, slight wrinkling of the polymeric layer is observed after 10 cycles, even though it maintained continuous contact with the active material. The ability of the polymer layer to bind to the surface of Si even after cycling contributes to the good cycling stability of the Si nanocomposite. Core-shell structures are not observed for the Si/DPA composites. Often capturing the formation of core-shell structure in a TEM (which is a product of lithiation) is dependent on stillness of the TEM column, therefore lack of a core-shell structure in imaging does not necessarily imply its absence [10].

Since, the individual Si particles did not fracture, the mechanical stability of the whole electrode is examined by placing it in the SEM. Fig. 7(a) depicts the surface of the nano Si electrode after just one cycle. It is seen that severe fracture takes place at the micro-level and large cracks on the surface of the electrode form islands with large gaps between them. Furthermore, EDS in Fig. 8(c), shows that the fracture penetrates the entire electrode thickness and reaches the current collector since a $\mathrm{Cu}$ signal is observed, whereas the same does not occur when Si is coated with DPA since in Fig. 8(d) a Cu signal from within the cracks was not detected. This assertion is further bolstered by the cross-sectional image of the electrode in 
the supplementary material (Fig. S5(a)) showing delamination from the current collector for the case when Si was not coated with DPA. Such severe instability after just one cycle leads to the significant capacity loss. Similar fracture is observed in the nano Si electrode after 50 cycles, but now the island (as measured across the diagonals) particles are smaller, as they continuously fragment during cycling. The average island decreases from $36.36 \mu \mathrm{m}$ after the $1^{\text {st }}$ cycle to $27.72 \mu \mathrm{m}$ after 50 cycles, a decrease of $24 \%$ over 50 cycles. The mean gap size between the islands during the $1^{\text {st }} 50$ cycles decreases from $4.5 \mu \mathrm{m}$ to $3.6 \mu \mathrm{m}$. This decrease in the gap size can be caused due to inability of electrode to de-lithiate upon losing electronic contact.

Similar cracking is observed for the Si/DPA electrode, however, the intensity and the depth of fracture is less severe. Comparing the morphology of the surface of the Si/DPA electrode Fig. 7(c-d) after the $1^{\text {st }}$ and $50^{\text {th }}$ cycles show that island size did not change much after initial fracture. Particularly, the average particle diagonal was reduced from $15.90 \mu \mathrm{m}$ after the $1^{\text {st }}$ cycle to $13.39 \mu \mathrm{m}$ after the $50^{\text {th }}$ cycle, a decrease of $15 \%$. The mean gap between the islands from the $1^{\text {st }}$ and $50^{\text {th }}$ cycle also did not change significantly as it increased from $1.58 \mu \mathrm{m}$ to $1.76 \mu \mathrm{m}$.

It can therefore be seen that even though the DPA coating could not prevent the initial cracking of the porous electrode, due to the large volumetric expansion of $\mathrm{Si}$, the polydopamine layer on the $\mathrm{Si}$ prevents reduction of the electrolyte on the Si. Such a rational strategy along with better adhesion within the electrode, allows for less strain on the SEI layer and hence it is more stable over longer cycling. Nonetheless, it is believed that formation of surface cracks is a benefit since they allow $\mathrm{Li}^{+}$to traverse deep within the electrode through the electrolyte. Moreover, a cross sectional image for the Si/DPA electrodes in Fig. S5(b) shows some delamination between the electrode and the current collector. However, the Si/DPA electrode itself appears to be unharmed and is not seen to pulverize or crumble after repeated cycling as the nano Si electrode does (Fig. S5(a)). In addition, the polydopamine layer on Si enhances the ionic conductivity $[52,53]$. 
The Si/DPA electrode maintains a better mechanical integrity since fracture did not reach the current collector, and less delamination occurred, allowing for a better electrochemical performance. This can be attributed to the continuous and uniform polymer layer on the Si active material which acts as a multifunctional protective layer as it enhances the mechanical stability of the porous electrode, since its multifunctional groups allow it to bind very well with both the Si surface on one side and the binder on the other, resulting in a more adherent composite. Moreover, it is well understood that DPA has high electrolyte uptake and enhances electrolyte wetting [53]. However, since the DPA completely coats the Si nanoparticles, it can be concluded that the direct contact between electrolyte and $\mathrm{Si}$ is diminished. This greatly decreases the room for fruition and reformation of the SEI layer on the surface of Si particle. As result a stable and high capacity retention is observed even at $0.2 \mathrm{C}\left(\sim 715 \mathrm{~mA} \mathrm{~g}^{-1}\right)$. Moreover, the SEM images show that the mechanical integrity of the Si/DPA electrode is maintained after 50 cycles with minimal damage being observed after the initial crack patterns formed in the $1^{\text {st }}$ cycle.

The increase in the C.E. (Fig. S7) and stable capacity retention of Fig. 5 can be explained by the formation of a stable SEI layer on the Si/DPA electrode. The decreased impedance of the Si/DPA electrode after 10 cycles in Fig. S6 bolsters the idea further. As does the fact that for the pure nano Si electrode, a higher impedance is obtained after 10 cycles (Fig. S6) suggesting the presence of an unstable SEI and hence more severe capacity decay. As can be seen in the bar graph in Fig. S7, the value of the C.E. for the Si and Si/DPA half cells shows a large difference in the favor of the Si/DPA electrode at the end of 5th and 10th cycle. Similar results are seen after the 20th, 50th and 75 th cycles. The higher walue of C.E. for the Si/DPA electrode further reveals the efficacy of the DPA layer. Specifically, a higher C.E. implies less irreversible consumption of $\mathrm{Li}^{+}$in the re(formation) of the SEI layer and hence it can be said that DPA allows for the formation of a more stable SEI.

It should be noted that in previous works the capacity for Si/DPA nanocomposites was $1800 \mathrm{mAh}$ $\mathrm{g}^{-1}$ and $1200 \mathrm{mAh} \mathrm{g}^{-1}[29,30,54]$, whereas our nanocomposite anodes herein show a superior performance of $2000 \mathrm{mAh} \mathrm{g}^{-1}$. This can be attributed to the fact that in fabricating the Si/DPA the concentration of the 
buffer solutions in the earlier works were lower, and polymerization was carried out for less time, which could result in a non-uniform DPA coating. However, no detailed microstructural analysis was done in $[29,30,54]$, and therefore this cannot be elaborated here in greater detail.

An initial illustration on the ability of DPA to increase the electrochemical stability of Si micron size particles is also shown in Fig. S8. Despite having a lower initial discharge capacity, the Si/DPA electrode retains a significantly higher capacity for thirty cycles, when compared to the uncoated $\mathrm{Si}$ microparticle electrodes. Such DPA coated Si microparticles will be examined in detail in a future study.

\section{Conclusion}

The present article compared the mechanical and electrochemical performance of porous electrodes which considered three cases as the active material (i) pure Si microparticles, (ii) Si nanoparticles, (iii) $\mathrm{Si}$ nanoparticles coated with polydopamine. A multiscale model was used to predict the mechanical stability of the Si micro and nanoparticles during lithiation, illustrating that at the microscale the particles fractured in half, whereas in the nanoscale no fracture occurred. Both of these results were in accordance with the experimental observations, which documented clearly the fracture in Si microparticles. To increase the cycling stability of $\mathrm{Si}$, we successfully used a facile single step polymerization technique to yield a $2 \mathrm{~nm}$ thick DPA layer on the Si nanoparticles. The synthesized composites resulted in a discharge capacity of $2000 \mathrm{mAh} \mathrm{g}^{-1}$ is observed after 100 cycles, which signifies a $15 \%$ increase in capacity retention over the pure nano Si electrode. The obtained Si/DPA nanocomposite benefitted from the polymer layer as it enhanced the mechanical its mechanical stability, unobstructed pathway for $\mathrm{Li}^{+}$and depressed continuous reformation of SEI layer. Additionally, encapsulation of many Si nanoparticle into clusters by the polymer reduces the actual surface area available for formation of the passivation layer, and EIS revealed a lower impedance due to the stable SEI layer. Even though individual Si particles did not fracture, in congruence with the model prediction, the capacity did fade with cycling for both the nano Si and Si/DPA electrodes. SEM images showed that this was attributed to fracture that occurred at the porous electrode level. 
Comparing SEM images of the whole porous cycled electrodes, with and without a DPA coating on the Si, showed that the cracks that formed in the porous electrode had a smaller width for the Si/DPA nanocomposites, alluding to better adhesion and mechanical integrity of the Si/DPA electrode. In concluding, initial results were presented which show that coating Si microparticles with DPA significantly improves the performance over uncoated Si microparticles. Use of larger scale Si particles in porous electrodes would greatly reduce cost and provide impetus for application in commercial devices. Herein the polymer and its choice perform a multifunctional role to increase mechanical integrity and also electrochemical performance of electrode.

\section{Acknowledgements}

The authors are grateful to the National Science Foundation for supporting this work through the CMMI grant [CMMI-1762602]. 


\section{REFERENCES}

[1] J. Li, J.R. Dahn, An In Situ X-Ray Diffraction Study of the Reaction of Li with Crystalline Si, J. Electrochem. Soc. 154 (2007) A156. https://doi.org/10.1149/1.2409862.

[2] L.Y. Beaulieu, K.W. Eberman, R.L. Turner, L.J. Krause, J.R. Dahn, Colossal Reversible Volume Changes in Lithium Alloys, (2001). https://doi.org/10.1149/1.1388178.

[3] M.N. Obrovac, L.J. Krause, Reversible Cycling of Crystalline Silicon Powder, J. Electrochem. Soc. 154 (2007) A103. https://doi.org/10.1149/1.2402112.

[4] V. Laokawee, N. Jarulertwattana, W. Susingrat, T. Sarakonsri, Synthesis of silicontin/nitrogen-doped reduced graphene oxide nanocomposite as anode materials for lithiumion batteries, in: Mater. Today Proc., Elsevier Ltd, 2019: pp. 1302-1308. https://doi.org/10.1016/j.matpr.2019.06.147.

[5] J. Graetz, C.C. Ahn, R. Yazami, B. Fultz, Highly reversible lithium storage in nanostructured silicon, Electrochem. Solid-State Lett. 6 (2003) A194. https://doi.org/10.1149/1.1596917.

[6] M. Holzapfel, H. Buqa, L.J. Hardwick, M. Hahn, A. Würsig, W. Scheifele, P. Novák, R. Kötz, C. Veit, F.M. Petrat, Nano silicon for lithium-ion batteries, Electrochim. Acta. 52 (2006) 973-978. https://doi.org/10.1016/j.electacta.2006.06.034.

[7] H. Li, X. Huang, L. Chen, Z. Wu, Y. Liang, A High Capacity Nano-Si Composite Anode Material for Lithium Rechargeable Batteries, 1999.

[8] L.Y. Beaulieu, K.W. Eberman, R.L. Turner, L.J. Krause, J.R. Dahna, Colossal reversible volume changes in lithium alloys, Electrochem. Solid-State Lett. 4 (2001) A137. https://doi.org/10.1149/1.1388178.

[9] X.H. Liu, L. Zhong, S. Huang, S.X. Mao, T. Zhu, J.Y. Huang, Size-dependent fracture of silicon nanoparticles during lithiation, ACS Nano. 6 (2012) 1522-1531.

https://doi.org/10.1021/nn204476h.

[10] M.T. McDowell, I. Ryu, S.W. Lee, C. Wang, W.D. Nix, Y. Cui, Studying the kinetics of crystalline silicon nanoparticle lithiation with in situ transmission electron microscopy, Adv. Mater. 24 (2012) 6034-6041. https://doi.org/10.1002/adma.201202744.

[11] X.H. Liu, J.Y. Huang, In situ TEM electrochemistry of anode materials in lithium ion batteries, Energy Environ. Sci. 4 (2011) 3844-3860. https://doi.org/10.1039/c1ee01918j.

[12] S. Kalnaus, K. Rhodes, C. Daniel, A study of lithium ion intercalation induced fracture of silicon particles used as anode material in Li-ion battery, J. Power Sources. 196 (2011) 8116-8124. https://doi.org/10.1016/j.jpowsour.2011.05.049.

[13] Z. Guo, L. Zhou, H. Yao, Improving the electrochemical performance of Si-based anode via gradient Si concentration, Mater. Des. 177 (2019) 107851. https://doi.org/10.1016/j.matdes.2019.107851.

[14] M. Winter, The solid electrolyte interphase - The most important and the least understood solid electrolyte in rechargeable Li batteries, Zeitschrift Fur Phys. Chemie. 223 (2009) 
1395-1406. https://doi.org/10.1524/zpch.2009.6086.

[15] H. Kim, M. Seo, M.H. Park, J. Cho, A critical size of silicon nano-anodes for lithium rechargeable batteries, Angew. Chemie - Int. Ed. 49 (2010) 2146-2149. https://doi.org/10.1002/anie.200906287.

[16] N. Liu, H. Wu, M.T. McDowell, Y. Yao, C. Wang, Y. Cui, A yolk-shell design for stabilized and scalable Li-ion battery alloy anodes, Nano Lett. (2012). https://doi.org/10.1021/nl3014814.

[17] R. Yi, F. Dai, M.L. Gordin, S. Chen, D. Wang, Micro-sized si-c composite with interconnected nanoscale building blocks as high-performance anodes for practical application in lithium-ion batteries, Adv. Energy Mater. 3 (2013) 295-300. https://doi.org/10.1002/aenm.201200857.

[18] M. Wu, J.E.C. Sabisch, X. Song, A.M. Minor, V.S. Battaglia, G. Liu, In situ formed Si nanoparticle network with micron-sized Si particles for lithium-ion battery anodes, Nano Lett. 13 (2013) 5397-5402. https://doi.org/10.1021/n1402953h.

[19] T. Munaoka, X. Yan, J. Lopez, J.W.F. To, J. Park, J.B.-H. Tok, Y. Cui, Z. Bao, Ionically Conductive Self-Healing Binder for Low Cost Si Microparticles Anodes in Li-Ion Batteries, Adv. Energy Mater. 8 (2018) 1703138. https://doi.org/10.1002/aenm.201703138.

[20] L. Wei, C. Chen, Z. Hou, H. Wei, Poly (acrylic acid sodium) grafted carboxymethyl cellulose as a high performance polymer binder for silicon anode in lithium ion batteries, Sci. Rep. 6 (2016) 1-8. https://doi.org/10.1038/srep19583.

[21] J. He, L. Zhang, Polyvinyl alcohol grafted poly (acrylic acid) as water-soluble binder with enhanced adhesion capability and electrochemical performances for Si anode, J. Alloys Compd. 763 (2018) 228-240. https://doi.org/10.1016/j.jallcom.2018.05.286.

[22] N.-S. Choi, S.-Y. Ha, Y. Lee, J.Y. Jang, M.-H. Jeong, W. Cheol Shin, M. Ue, Recent Progress on Polymeric Binders for Silicon Anodes in Lithium-Ion Batteries, J. Electrochem. Sci. Technol. 6 (2015) 35. https://doi.org/10.5229/JECST.2015.6.2.35.

[23] J. Song, M. Zhou, R. Yi, T. Xu, M.L. Gordin, D. Tang, Z. Yu, M. Regula, D. Wang, Interpenetrated Gel Polymer Binder for High-Performance Silicon Anodes in Lithium-ion Batteries, Adv. Funct. Mater. 24 (2014) 5904-5910. https://doi.org/10.1002/adfm.201401269.

[24] J. Zhang, S. Fan, H. Wang, J. Qian, H. Yang, X. Ai, J. Liu, Surface-Bound Silicon Nanoparticles with a Planar-Oriented N-Type Polymer for Cycle-Stable Li-Ion Battery Anode, ACS Appl. Mater. Interfaces. 11 (2019) 13251-13256. https://doi.org/10.1021/acsami.9b00939.

[25] X. Zhao, M. Li, N. Ross, Y.M. Lin, Towards cost-effective silicon anodes using conductive polyaniline-encapsulated silicon micropowders, RSC Adv. 6 (2016) 77682 77687. https://doi.org/10.1039/c6ra14386e.

[26] S.Y. Chew, Z.P. Guo, J.Z. Wang, J. Chen, P. Munroe, S.H. Ng, L. Zhao, H.K. Liu, Novel nano-silicon/polypyrrole composites for lithium storage, Electrochem. Commun. 9 (2007) 
941-946. https://doi.org/10.1016/j.elecom.2006.11.028.

[27] Z.F. Li, H. Zhang, Q. Liu, Y. Liu, L. Stanciu, J. Xie, Novel pyrolyzed polyaniline-grafted silicon nanoparticles encapsulated in graphene sheets as Li-ion battery anodes, ACS Appl. Mater. Interfaces. 6 (2014) 5996-6002. https://doi.org/10.1021/am501239r.

[28] M. Tian, P. Wu, Nature Plant Polyphenol Coating Silicon Submicroparticle Conjugated with Polyacrylic Acid for Achieving a High-Performance Anode of Lithium-Ion Battery, ACS Appl. Energy Mater. 2 (2019) 5066-5073. https://doi.org/10.1021/acsaem.9b00734.

[29] Y. Bie, J. Yang, X. Liu, J. Wang, Y. Nuli, W. Lu, Polydopamine wrapping silicon crosslinked with polyacrylic acid as high-performance anode for lithium-ion batteries, ACS Appl. Mater. Interfaces. 8 (2016) 2899-2904. https://doi.org/10.1021/acsami.5b10616.

[30] C. Fang, Y. Deng, Y. Xie, J. Su, G. Chen, Improving the electrochemical performance of Si nanoparticle anode material by synergistic strategies of polydopamine and graphene oxide coatings, J. Phys. Chem. C. 119 (2015) 1720-1728.

https://doi.org/10.1021/jp511179s.

[31] E. Bohn, T. Eckl, M. Kamlah, R. McMeeking, A Model for Lithium Diffusion and Stress Generation in an Intercalation Storage Particle with Phase Change, J. Electrochem. Soc. 160 (2013) A1638-A1652. https://doi.org/10.1149/2.011310jes.

[32] S. Huang, F. Fan, J. Li, S. Zhang, T. Zhu, Stress generation during lithiation of highcapacity electrode particles in lithium ion batteries, Acta Mater. 61 (2013) 4354-4364. https://doi.org/10.1016/j.actamat.2013.04.007.

[33] L. Chen, F. Fan, L. Hong, J. Chen, Y.Z. Ji, S.L. Zhang, T. Zhu, L.Q. Chen, JES FOCUS ISSUE ON MECHANO-ELECTRO-CHEMICAL COUPLING IN ENERGY RELATED MATERIALS AND DEVICES A Phase-Field Model Coupled with Large Elasto-Plastic Deformation: Application to Lithiated Silicon Electrodes, J. Electrochem. Soc. 161 (2014) 3164-3172. https://doi.org/10.1149/2.0171411jes.

[34] M. Klinsmann, D. Rosato, M. Kamlah, R.M. McMeeking, Modeling Crack Growth during Li Extraction in Storage Particles Using a Fracture Phase Field Approach, J. Electrochem. Soc. 163 (2016) A102-A118. https://doi.org/10.1149/2.0281602jes.

[35] C. Miehe, H. Dal, L.-M. Schänzel, A. Raina, A phase-field model for chemo-mechanical induced fracture in lithium-ion battery electrode particles, Int. J. Numer. Methods Eng. 106 (2016) 683-711. https://doi.org/10.1002/nme.5133.

[36] J. Réthoré, H. Zheng, H. Li, J. Li, K.E. Aifantis, A multiphysics model that can capture crack patterns in Si thin films based on their microstructure, J. Power Sources. 400 (2018) 383-391. https://doi.org/10.1016/j.jpowsour.2018.07.106.

[37] R.A. Sharma, Thermodynamic Properties of the Lithium-Silicon System, J. Electrochem. Soc. 123 (1976) 1763. https://doi.org/10.1149/1.2132692.

[38] B.A. Boukamp, G.C. Lesh, R.A. Huggins, J.E. Soc, All - Solid Lithium Electrodes with Mixed - Conductor Matrix Tetra Acknowledgment All-Solid Lithium Electrodes with Mixed-Conductor Matrix, Joutnal Electrochem. Soc. 128 (1981) 725-729. https://doi.org/10.1149/1.2127495. 
[39] Y. Kubota, M.C.S. Escao, H. Nakanishi, H. Kasai, Crystal and electronic structure of Li15Si4, J. Appl. Phys. 102 (2007) 53704. https://doi.org/10.1063/1.2775999.

[40] E.K. Rahani, V.B. Shenoy, Role of Plastic Deformation of Binder on Stress Evolution during Charging and Discharging in Lithium-Ion Battery Negative Electrodes, J. Electrochem. Soc. 160 (2013) A1153-A1162. https://doi.org/10.1149/2.046308jes.

[41] H.S. Kim, D.W. Lee, D.H. Kim, D.S. Kong, J. Choi, M. Lee, G. Murillo, J.H. Jung, Dominant role of young's modulus for electric power generation in PVDF-BaTiO3 composite-based piezoelectric nanogenerator, Nanomaterials. 8 (2018). https://doi.org/10.3390/nano8100777.

[42] Y.S. Choi, M. Pharr, K.H. Oh, J.J. Vlassak, A simple technique for measuring the fracture energy of lithiated thin-film silicon electrodes at various lithium concentrations, J. Power Sources. 294 (2015) 159-166. https://doi.org/10.1016/j.jpowsour.2015.06.063.

[43] N. Delpuech, D. Mazouzi, N. Dupré, P. Moreau, M. Cerbelaud, J.S. Bridel, J.C. Badot, E. De Vito, D. Guyomard, B. Lestriez, B. Humbert, Critical role of silicon nanoparticles surface on lithium cell electrochemical performance analyzed by FTIR, Raman, EELS, XPS, NMR, and BDS spectroscopies, J. Phys. Chem. C. 118 (2014) 17318-17331. https://doi.org/10.1021/jp503949y.

[44] D.R. Dreyer, D.J. Miller, B.D. Freeman, D.R. Paul, C.W. Bielawski, Elucidating the structure of poly(dopamine), Langmuir. 28 (2012) 6428-6435. https://doi.org/10.1021/la204831b.

[45] R.A. Zangmeister, T.A. Morris, M.J. Tarlov, Characterization of Polydopamine Thin Films Deposited at Short Times by Autoxidation of Dopamine, 29 (2013) 26. https://doi.org/10.1021/la400587j.

[46] C.C. Ho, S.J. Ding, Structure, properties and applications of mussel-inspired polydopamine, J. Biomed. Nanotechnol. 10 (2014) 3063-3084. https://doi.org/10.1166/jbn.2014.1888.

[47] R. Batul, M. Bhave, P.J. Mahon, A. Yu, Polydopamine nanosphere with in-situ loaded gentamicin and its antimicrobial activity, Molecules. 25 (2020) 2090. https://doi.org/10.3390/molecules25092090.

[48] D.B. Mawhinney, J.A. Glass, J.T. Yates, FTIR study of the oxidation of porous silicon, J. Phys. Chem. B. 101 (1997) 1202-1206. https://doi.org/10.1021/jp963322r.

[49] M. Schnabel, E. Arca, Y. Ha, C. Stetson, G. Teeter, S.D. Han, P. Stradins, Enhanced Interfacial Stability of $\mathrm{Si}$ Anodes for Li-Ion Batteries via Surface SiO2Coating, ACS Appl. Energy Mater. 3 (2020) 8842-8849. https://doi.org/10.1021/acsaem.0c01337.

[50] J. Wei Wang, Y. He, F. Fan, X. Hua Liu, S. Xia, Y. Liu, C. Thomas Harris, H. Li, J. Yu Huang, S.X. Mao, T. Zhu, Two-Phase Electrochemical Lithiation in Amorphous Silicon, Nano Lett. 13 (2013). https://doi.org/10.1021/nl304379k.

[51] X.H. Liu, F. Fan, H. Yang, S. Zhang, J.Y. Huang, T. Zhu, Self-limiting lithiation in silicon nanowires, ACS Nano. 7 (2013) 1495-1503. https://doi.org/10.1021/nn305282d. 
[52] M.H. Ryou, Y.M. Lee, J.K. Park, J.W. Choi, Mussel-inspired polydopamine-treated polyethylene separators for high-power Li-ion batteries, Adv. Mater. 23 (2011) 30663070. https://doi.org/10.1002/adma.201100303.

[53] L. Wang, Z. Dong, D. Wang, F. Zhang, J. Jin, Covalent bond glued sulfur nanosheetbased cathode integration for long-cycle-life Li-S batteries, Nano Lett. 13 (2013) 62446250. https://doi.org/10.1021/nl403715h.

[54] D. Song, D. Jung, I. Cho, M.-H. Ryou, Y.M. Lee, Mussel-Inspired PolydopamineFunctionalized Super-P as a Conductive Additive for High-Performance Silicon Anodes, Adv. Mater. Interfaces. 3 (2016) 1600270. https://doi.org/10.1002/admi.201600270. 

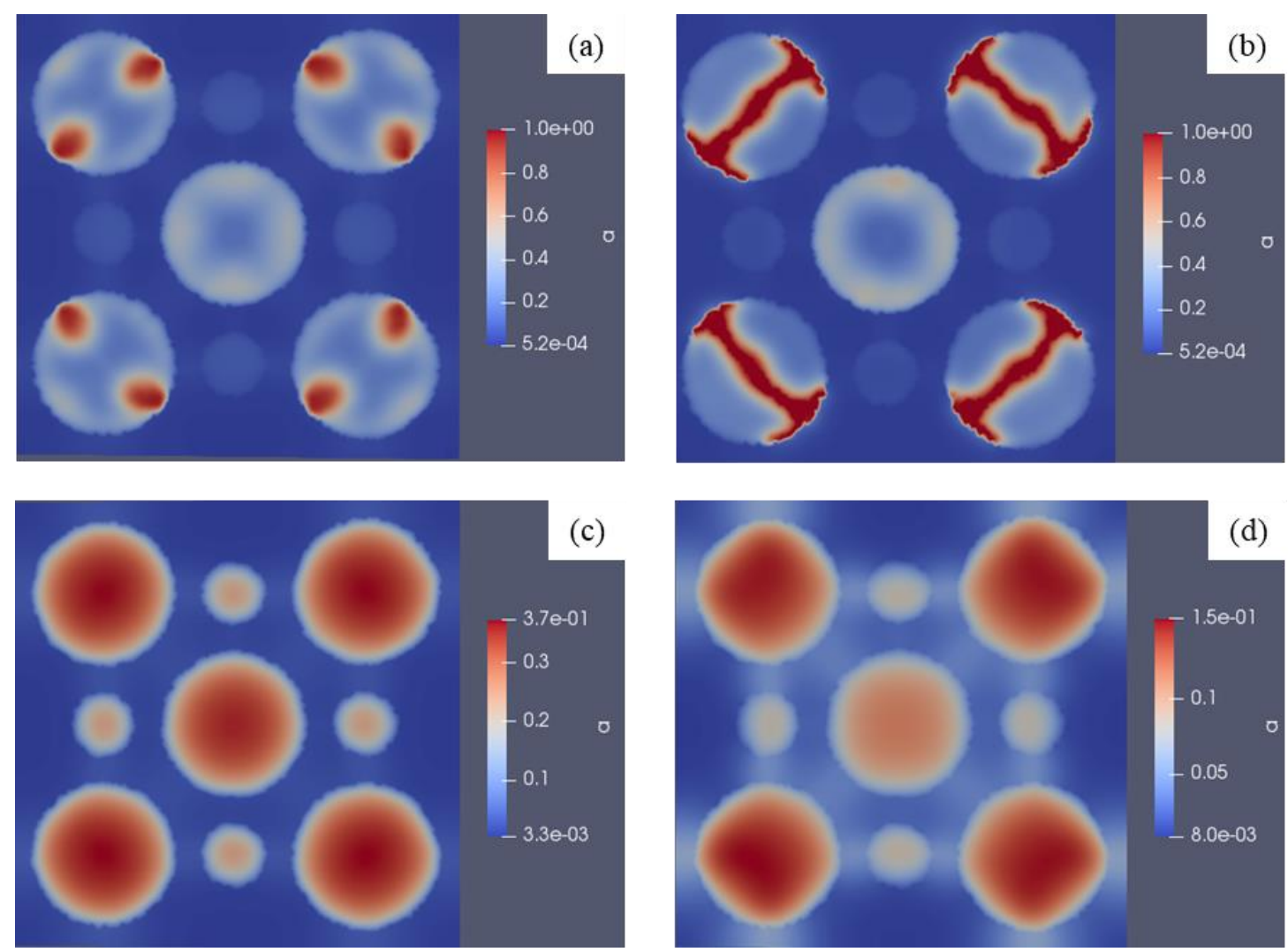

Figure 1 Damage profile of Si particles with diameter of $0.95 \mu$ m after (a) full insertion and (b) de-insertion. Damage profile of Si particle with diameter of $95 \mathrm{~nm}$ after (c) full insertion and (d) de-insertion
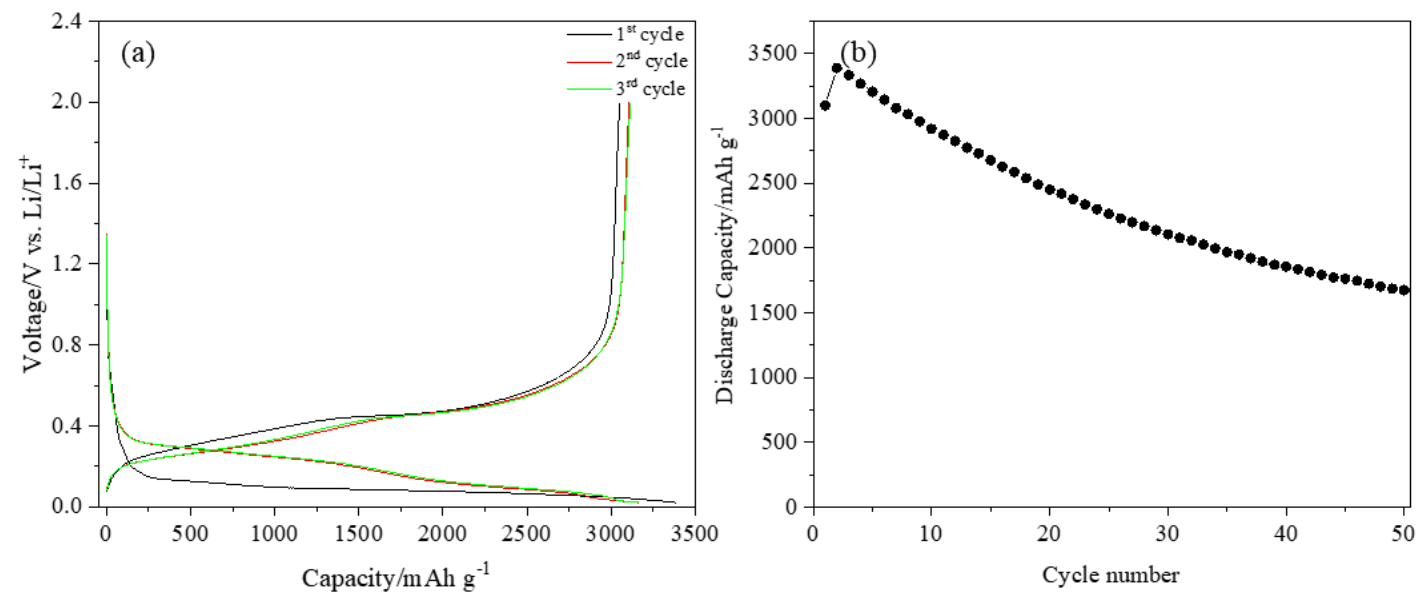

Figure 2 Microscale Si electrode: (a) Initial voltage profile and (b) cycling performance at $0.2 C$ 

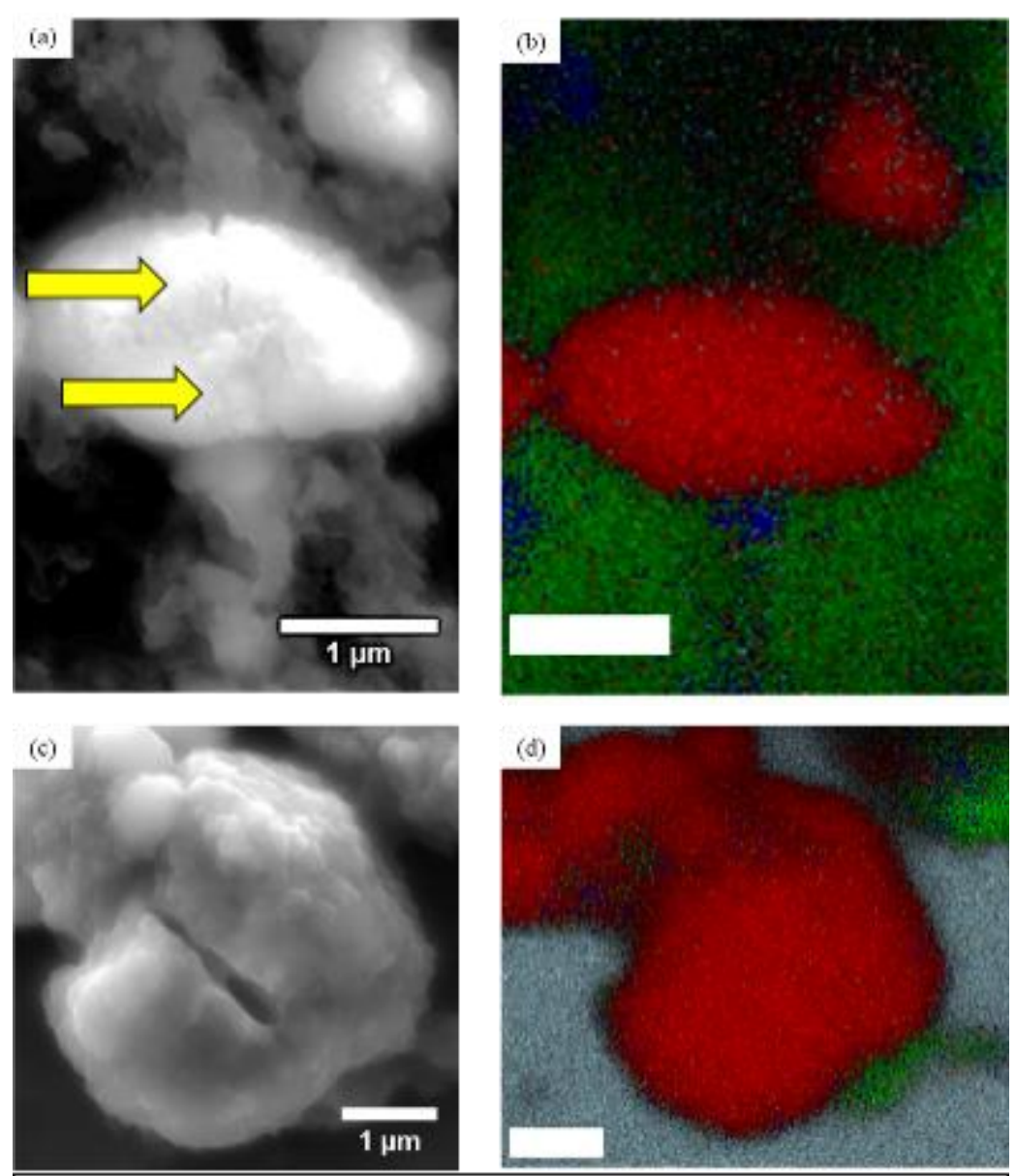

(d)

Red: Silium, Green: Canbum, Blue Oxygen

Figure 3 Microscale Si electrode after 1 cycle (a) SEM image, (b) EDS map. After 2 cycles (c) SEM image, (d) EDS map (scale bar 1 $\mu \mathrm{m})$ 


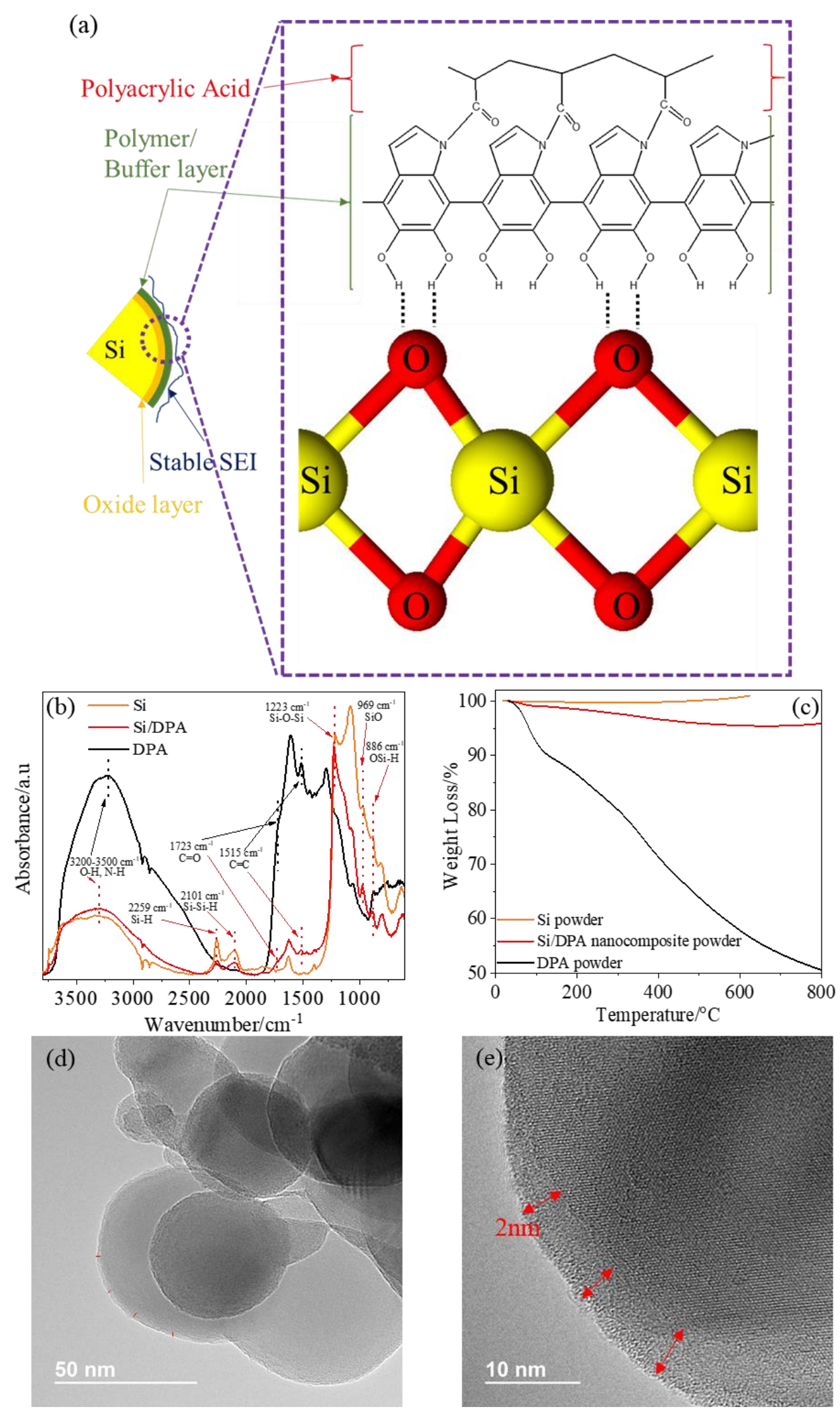

Figure 4 (a) Illustration of Si/DPA nanocomposite, (b) FT-IR of Si/DPA nanocomposite, Si, polydopamine polymer (c) TGA of Si/DPA nanocomposite, Si, polydopamine polymer, (d-e) TEM images of Si/DPA nanocomposite. 

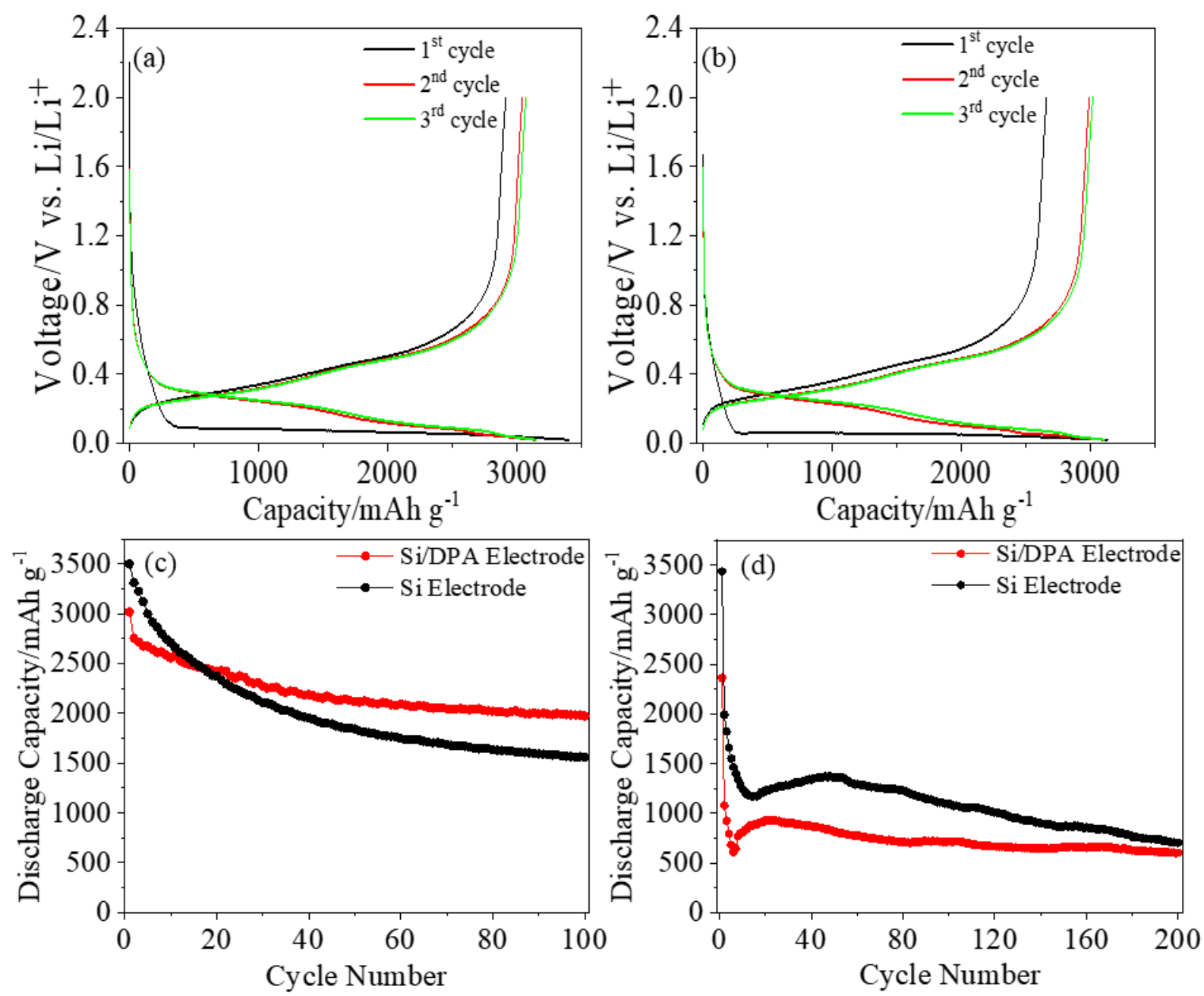

Figure 5 Initial voltage profile of (a) nano Si electrode, (b) Si/DPA electrode. Cycling performance of nano Si and Si/DPA electrode (c) at $0.2 C$ and (d) $1 C$ 

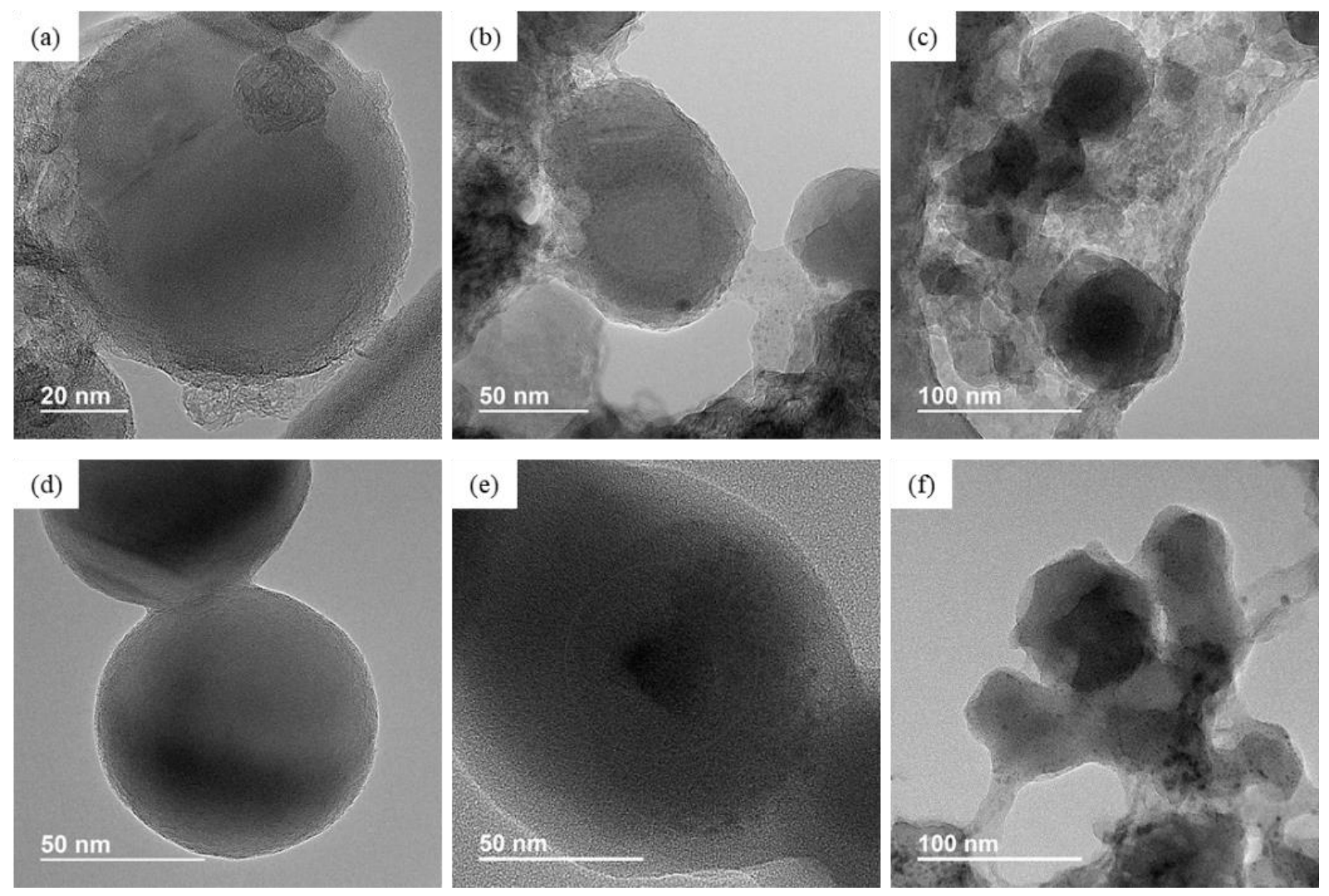

Figure 6 TEM image of (a) Pristine nano Si electrode, (b) nano Si electrode after 1 cycle, (c) nano Si electrode after 10 cycles; (d) Pristine Si/DPA electrode, (e) Si/DPA electrode after $1 \mathrm{cycle}$, (f) Si/DPA electrode after 10 cycles 

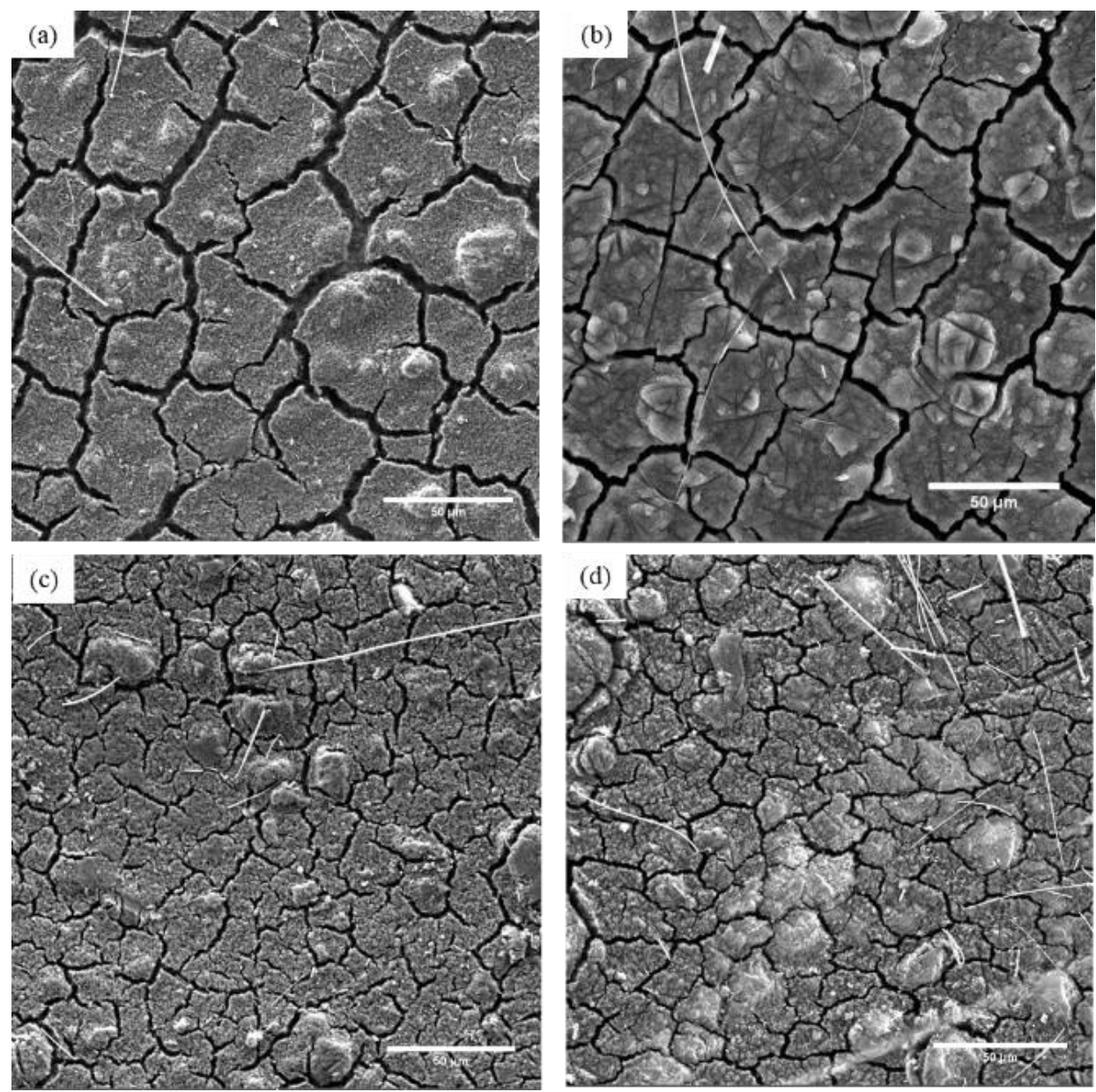

Figure 7 SEM images of (a) nano Si electrode after 1 cycle, (b) nano Si electrode after 50 cycles, (c) Si/DPA electrode after 1 cycle, (d) Si/DPA electrode after 50 cycles. (Scale bar $50 \mu \mathrm{m}$ ) 

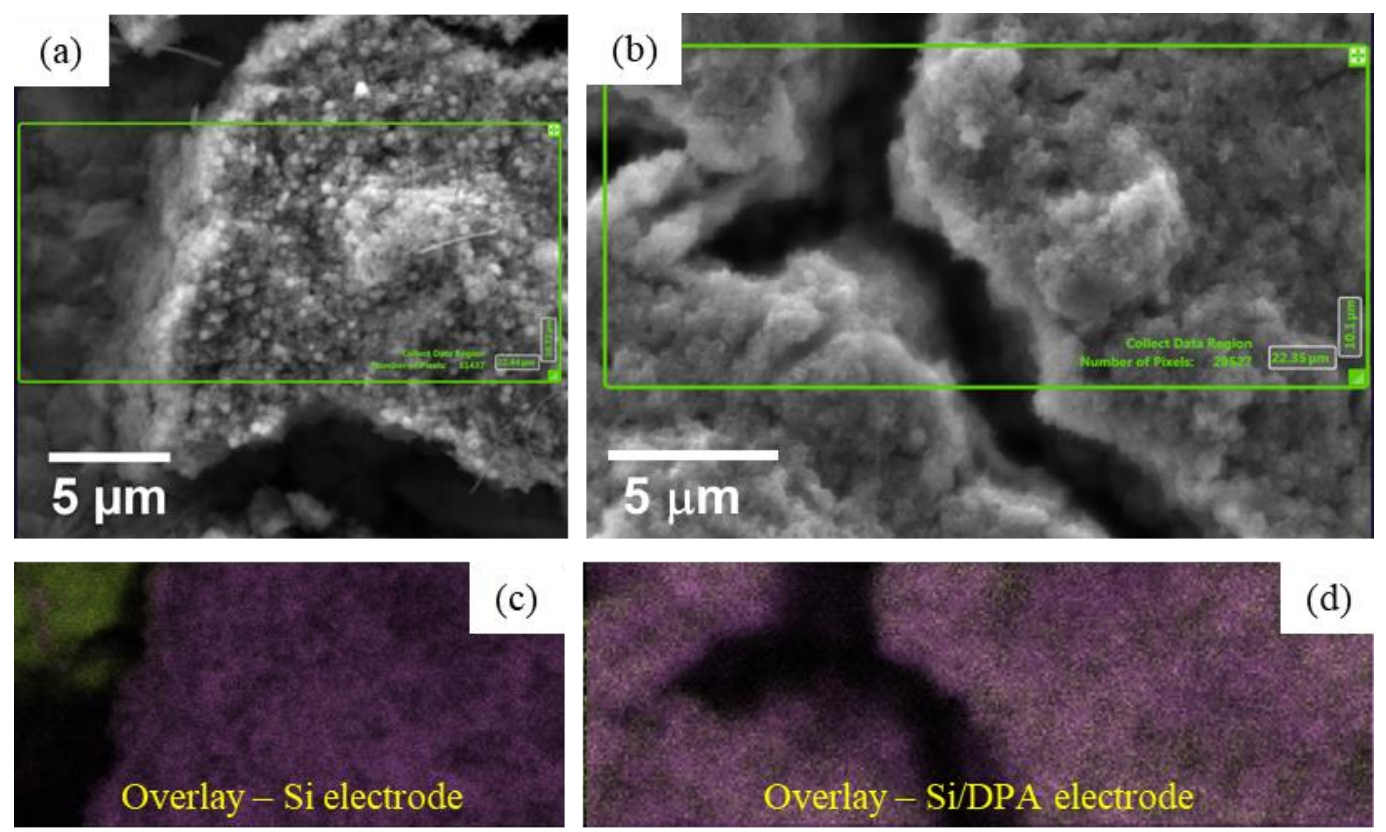

Figure 8 (a) SEM image of Si electrode after 1 cycle, (b) SEM image of Si/DPA electrode after 1 cycle. (c) EDS Elemental map showing overlay of Si (pink) and Cu (green) in Si electrode and (d) ) EDS Elemental map showing overlay of Si (pink) and Cu (green) in Si electrode Si/DPA electrode. 